\title{
miR-5591-5p regulates the effect of ADSCs in repairing diabetic wound via targeting AGEs/AGER/JNK signaling axis
}

\author{
Qiang Li', Sizhan Xia', Yating Yin', Yanping Guo', Feifei Chen ${ }^{2}$ and Peisheng Jin $^{1,2}$
}

\begin{abstract}
Advanced glycation end products/advanced glycation end products receptor (AGEs/AGER) interaction triggers reactive oxygen species (ROS) generation and activates downstream signal pathways and induces apoptosis in endothelial progenitor cells. A number of studies have revealed the involvement of microRNAs (miRNAs) in regulating intracellular ROS production and apoptosis. However, few studies explore the role of miRNAs in regulating the effect of adipose tissue-derived stem cells (ADSCs) in repairing diabetic wound and the associated cellular mechanisms remain unclear. In this study, ADSCs were exposed to AGEs, then siRNA for AGER was transfected into ADSCs. We found that AGEs/AGER axis induced ROS generation and apoptosis in ADSCs. AGEs treatment downregulated miR-5591-5p in ADSCs, which directly targeted AGER. miR-5591-5p suppressed AGEs/AGER axis-mediated ROS generation and apoptosis in ADSCs in vitro. In addition, miR-5591-5p promoted cell survival and enhanced the ability of ADSCs for repairing cutaneous wound in vivo. Furthermore, we confirmed that c-jun kinase (JNK) signal was involved in the inhibitory effect of miR-5591-5p on AGEs/AGER axis-induced ROS generation and apoptosis in ADSCs. Thus, these results indicated that miR-5591-5p targeting AGES/AGER/JNK signaling axis possibly regulates the effect of ADSCs in repairing diabetic wound.
\end{abstract}

\section{Introduction}

Adipose tissue-derived stem cells (ADSCs) are derived from adipose tissue stroma, which harbor the ability of self-renewal and differentiate into a number of functional cells ${ }^{1}$. Emerging evidence has shown the beneficial effects of ADSCs administration to treat various diseases because of their simple isolation techniques, easy expandability, low immunogenicity, and pluripotency ${ }^{2}, 3$. Moreover, ADSCs have been found to promote chronic wound healing ${ }^{4}$. Diabetic patients are much more susceptible to developing chronic wounds. ADSCs therapy could potentially influence the treatment of wounds in non-

Correspondence: Feifei Chen (chenfeifei@xzhmu.edu.cn) or

Peisheng Jin (100000401006@xzhmu.edu.cn)

${ }^{1}$ Department of Plastic Surgery, Affiliated Hospital of Xuzhou Medical

University, Jiangsu, Xuzhou, China

${ }^{2}$ Jiangsu Center for the Collaboration and Innovation of Cancer, Xuzhou

Medical University, Jiangsu, Xuzhou, China

These authors contributed equally: Qiang Li, Sizhan Xia, Yating Yin.

Edited by Y Shi diabetic conditions, but no effect in diabetic patients ${ }^{5}$. Increased apoptosis in stem cells has been considered to impair wound healing in a diabetic rat model ${ }^{6}$. Although efforts has been made to improve cell survival after implantation, more simple and efficient interventions by which to protect ADSCs against apoptosis and increase the therapeutic effect of ADSCs are still required.

Advanced glycation end products (AGEs) refer to a group of heterogeneous macromolecules that are produced by the post-translational modification of proteins via non-enzymatic glycation, lipids and nucleic acids, accumulate with age, and are abundantly elevated in diabetic patients ${ }^{7}$. The increased AGEs in diabetic patients cause a number of pathological changes. There has been evidence that elevated AGEs promotes apoptosis of endothelial progenitor cell (EPC) and endothelial cell inhibits proliferation of repairing cells, thus impedes wound healing ${ }^{8-10}$. Numerous articles have documented

\section{(c) The Author(s) 2018}

(c) (i) Open Access This article is licensed under a Creative Commons Attribution 4.0 International License, which permits use, sharing, adaptation, distribution and reproduction cc) in any medium or format, as long as you give appropriate credit to the original author(s) and the source, provide a link to the Creative Commons license, and indicate if changes were made. The images or other third party material in this article are included in the article's Creative Commons license, unless indicated otherwise in a credit line to the material. If material is not included in the article's Creative Commons license and your intended use is not permitted by statutory regulation or exceeds the permitted use, you will need to obtain permission directly from the copyright holder. To view a copy of this license, visit http://creativecommons.org/licenses/by/4.0/. 


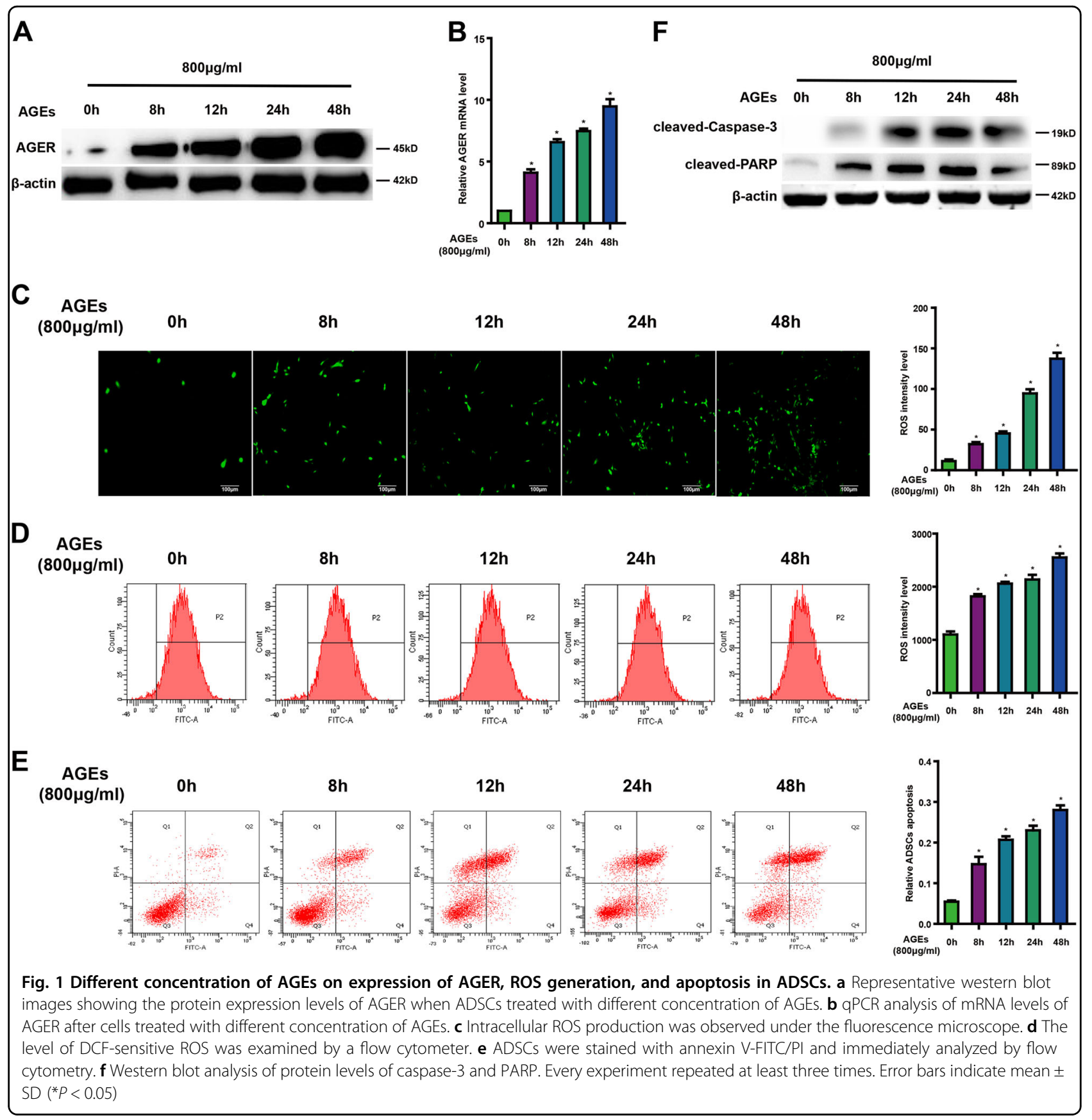

that AGEs induce cell apoptosis and may involve in the pathogenesis of biophysical disorders ${ }^{11,12}$. AGEs provide the bridge between intracellular and extracellular damage through the specific receptor for advanced glycation end products receptor (AGER). AGER is a $45-\mathrm{kDa}$ transmembrane receptor, which belongs to the immunoglobulin superfamily. It is found to be expressed highly during embryonic development, but less expressed in adult tissues ${ }^{13}$. However, pathological conditions of high glucose, reactive oxygen species (ROS), hypoxia, proinflammatory mediators, or AGEs itself induce AGER expression $^{13,14}$. AGE/AGER interactions lead to a diverse array of signaling pathways activation, such as p38 and JNK that participate in apoptosis ${ }^{15,16}$.

MicroRNAs (miRNAs) are ubiquitously expressed short non-coding RNAs of 20-22 nucleotides, which regulate messenger RNAs (mRNAs) after transcription by targeting the untranslated regions. This leads to degradation of the target mRNAs and/or translation inhibition ${ }^{17}$. Recently, several miRNAs have been demonstrated to interfere with and modulate intracellular apoptosis signaling ${ }^{18,} 19$. However, few studies explore the role of 
miRNAs in AGEs/AGER signaling related to diabetic wound healing. In this study, we focused on miR-5591-5p via a miRNA array after ADSCs exposure to AGEs. Then, we focused on the role of miR-5591-5p in ADSCs exposed to AGEs, and found that miR-5591-5p regulated the effect of ADSCs in repairing diabetic wound healing via targeting AGES/AGER/JNK signaling axis.

\section{Results}

AGEs induces AGER expression, ROS generation, and cell apoptosis in ADSCs

The cellular effects of AGEs are mainly mediated through the receptor for AGEs. To investigate whether AGEs affect the expression of AGER in ADSCs, cells were incubated with or without AGEs $(100-1600 \mu \mathrm{g} / \mathrm{ml})$ for 24 $h$, the expression of AGER was established by western blot and quantitative PCR. As shown in Figure 1a, b, the upregulation of AGER in response to AGEs was identified in a dose-dependent manner. Fluorescence microscope and flow cytometer showed that production of ROS was increased after AGEs treatment (Fig. 1c, d). In addition, data from flow cytometer displayed that apoptotic cells were increased with AGEs (Fig. 1e). Caspase-3 and PARP are the principal apoptosis markers through which the mitochondrial and cytosolic pathways induce apoptosis. Consequently, we examined the activity of caspase- 3 and PARP. Western blot analysis indicated that AGEs treatment promoted caspase-3 and PARP activity (Fig. 1f).

Moreover, expression of AGER in ADSCs following treatment with $800 \mu \mathrm{g} / \mathrm{ml}$ AGEs was determined at the different time points. Our results displayed a obvious increase of AGER from 0 to $48 \mathrm{~h}$ (Fig. 2a, b). At the same time, ROS level showed distinct elevation from 0 to $48 \mathrm{~h}$ in Figure 2c, which is consistent with the time course of enhanced fluorescence probe expression treated with AGEs in Figure 2d. The apoptotic cells were increased in a time course after given AGEs (Fig. 2e). The upregulation of cleaved-caspase-3 and PARP in response to AGEs was also shown in a time-dependent manner (Fig. 2f).

\section{AGEs/AGER axis induces ROS generation and apoptosis in ADSCs}

AGEs can activate diverse signal transduction cascades and downstream pathways, including generation of ROS, leading to apoptosis. To investigate whether AGER is essential for the role of AGEs in ADSCs, we first detected AGER protein and mRNA levels after using smallinterfering RNAs (siRNAs) for AGER. The data showed that AGER was enhanced after exposure to AGEs, but declined significantly after transfected with siRNAs for AGER (Fig. 3a, b). Fluorescence microscope showed that production of ROS was increased after AGEs treatment, but diminished after knockdown of AGER, which was consistent with the results of flow cytometer (Fig. 3c, d).
Furthermore, we explored whether AGEs/AGER was involved in the regulation of apoptosis in ADSCs. Flow cytometer showed that AGEs induced apoptosis, but this was reversed by inhibition of AGER expression (Fig. 3e). Western blot assay indicated that siRNAs for AGER prevented caspase- 3 and PARP from cleavage to suppress caspase signal pathway (Fig. 3f). These data revealed that AGEs/AGER axis induced ROS generation and apoptosis in ADSCs.

\section{AGER is a direct target of miR-5591-5p}

Because miRNAs have been reported to regulate intracellular ROS production and apoptosis signaling in endothelial cells during diabetes ${ }^{20,21}$, we used a miRNA array to analyze the miRNA profiles in AGEs-treated ADSCs and control cells. The data showed a large number of miRNAs that were downregulated in AGEs-treated ADSCs (Fig. 4a). We then employed miRDB and targetscan softwares to analyze these differentially expressed miRNAs. Only miR-5591-5p was found to be partially complementary to the conserved site in the 3 '-untranslated region (UTR) of AGER mRNA (Fig. 4b). To confirm whether miR-5591-5p targets AGER mRNA, we cloned the $3^{\prime}$-UTR sequence of AGER into the psiCHECK ${ }^{\mathrm{Tx}}-2$ vector. The data showed that introduction of miR-5591$5 \mathrm{p}$ diminished luciferase activity of this reporter. In order to verify whether miR-5591-5p specifically inhibited AGER through its potential binding site, we constructed the mutated (mutant type, MUT) reporter at miR-5591$5 p$-binding site. Our data showed that activity of the MUT 3'-UTR reporter was unchanged (Fig. 4c, d). Moreover, we evaluated the effects of miR-5591-5p on AGER mRNA and protein levels in ADSCs by quantitative PCR (qPCR) and western blot. We found that transfection of miR5591-5p mimics into ADSCs negatively regulated AGER expression in ADSCs (Fig. 4e, f). Taken together, these findings suggested that miR-5591-5p could directly target the 3'-UTR of AGER and downregulate AGER expression.

miR-5591-5p suppresses AGEs/AGER axis-mediated ROS generation and apoptosis in ADSCs

Because AGER is a target of miR-5591-5p, we further verified whether miR-5591-5p regulated ROS generation and cell apoptosis induced by AGEs/AGER. For the ROS generation, the data showed that miR-5591-5p diminished production of ROS, which was induced by AGEs (Fig. $5 \mathrm{a}-\mathrm{d}$ ). In addition, data of flow cytometer showed that miR-5591-5p also suppressed AGEs-induced apoptosis (Fig. 5e). Western blot analysis for cleaved-caspase-3 and PARP suggested that transfected miR-5591-5p mimics inhibited caspase-3 and PARP activity (Fig. 5f). Conversely, miR-5591-5p inhibitors promoted ROS production induced by AGEs (Fig. 5g-j). Flow cytometer showed that miR-5591-5p inhibitors facilitated AGEs- 


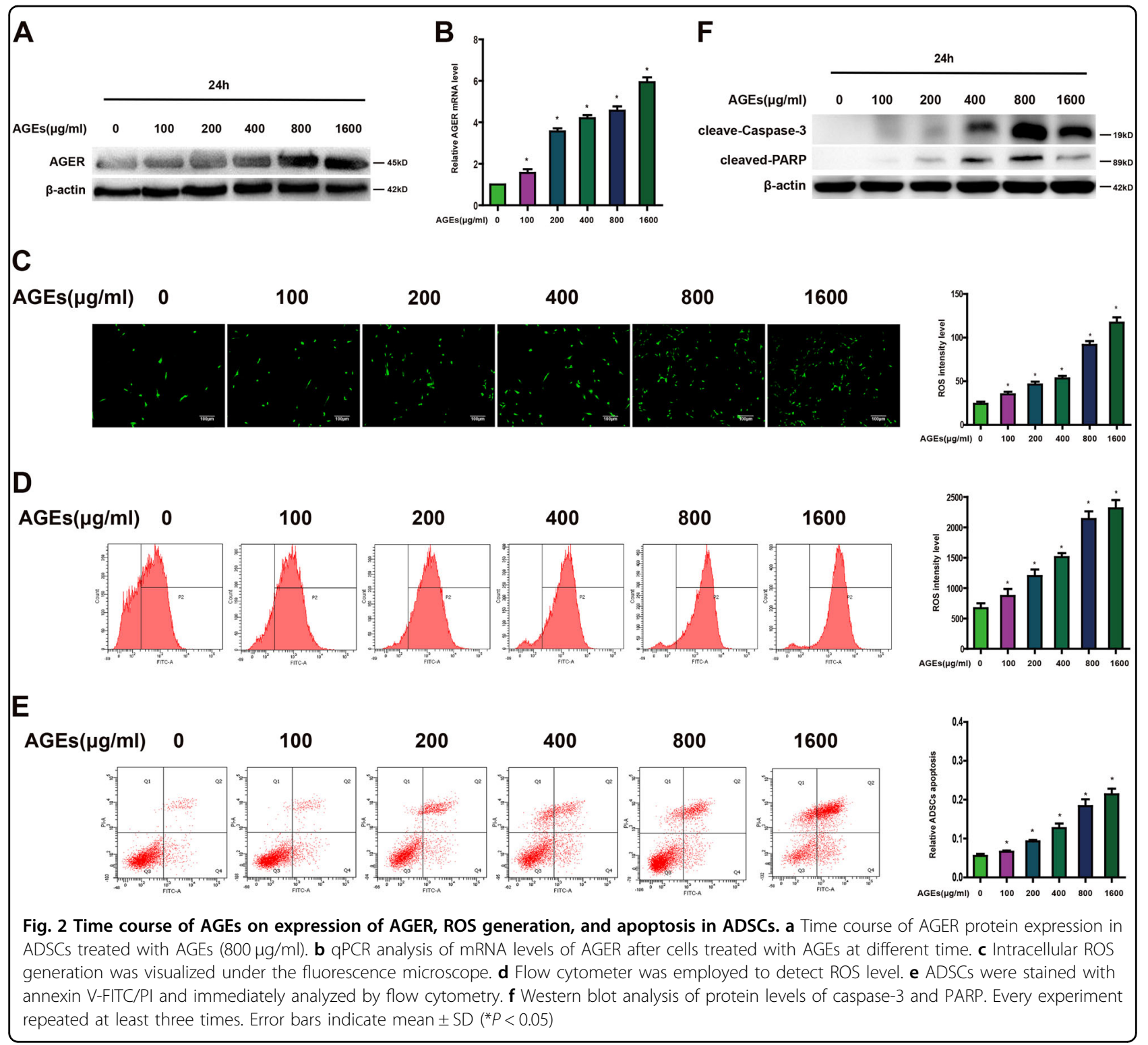

induced apoptosis (Fig. 5k). The activity of caspase-3 and PARP was elevated by miR-5591-5p inhibitors (Fig. 5l). When we co-transfected vector of AGER and miR-5591$5 \mathrm{p}$ mimics in ADSCs, data from supplementary Figure 1 showed that overexpression of AGER promoted ROS generation and apoptosis of ADSCs. However, this positive effect of AGER on ROS generation and apoptosis can be suppressed by miR-5591-5p mimics in ADSCs. These results indicated that miR-5591-5p may act as a negative modulator of AGEs/AGER-induced apoptosis in ADSCs.

Phosphorylation of JNK signal is involved in AGEs/AGERinduced ROS generation and apoptosis in ADSCs

To investigate the potential downstream pathway that regulated by AGEs/AGER, we performed KEGG pathway analysis to search for the potential signal pathway that regulated by AGES. The top 10 KEGG pathways enriched for the AGEs targets were displayed in Figure 6a. Previous studies have reported that the MAPK pathway is critical for production of ROS and induction of apoptosis, we asked if AGEs/AGER axis triggers the MAPK pathway in ADSCs. We first detected the phosphorylation of MAPK family members (ERK1/2, JNK, and p38 MAPK) after treated with AGEs. As shown in Figure 6b, exposure to AGEs significantly increased JNK activation in a timedependent manner, but no change of ERK1/2 and p38 phosphorylation.

To further determine whether JNK pathway is activated by AGEs/AGER axis, ADSCs transfected with siRNA for AGER were treated with JNK activator, anisomycin $(800$ 


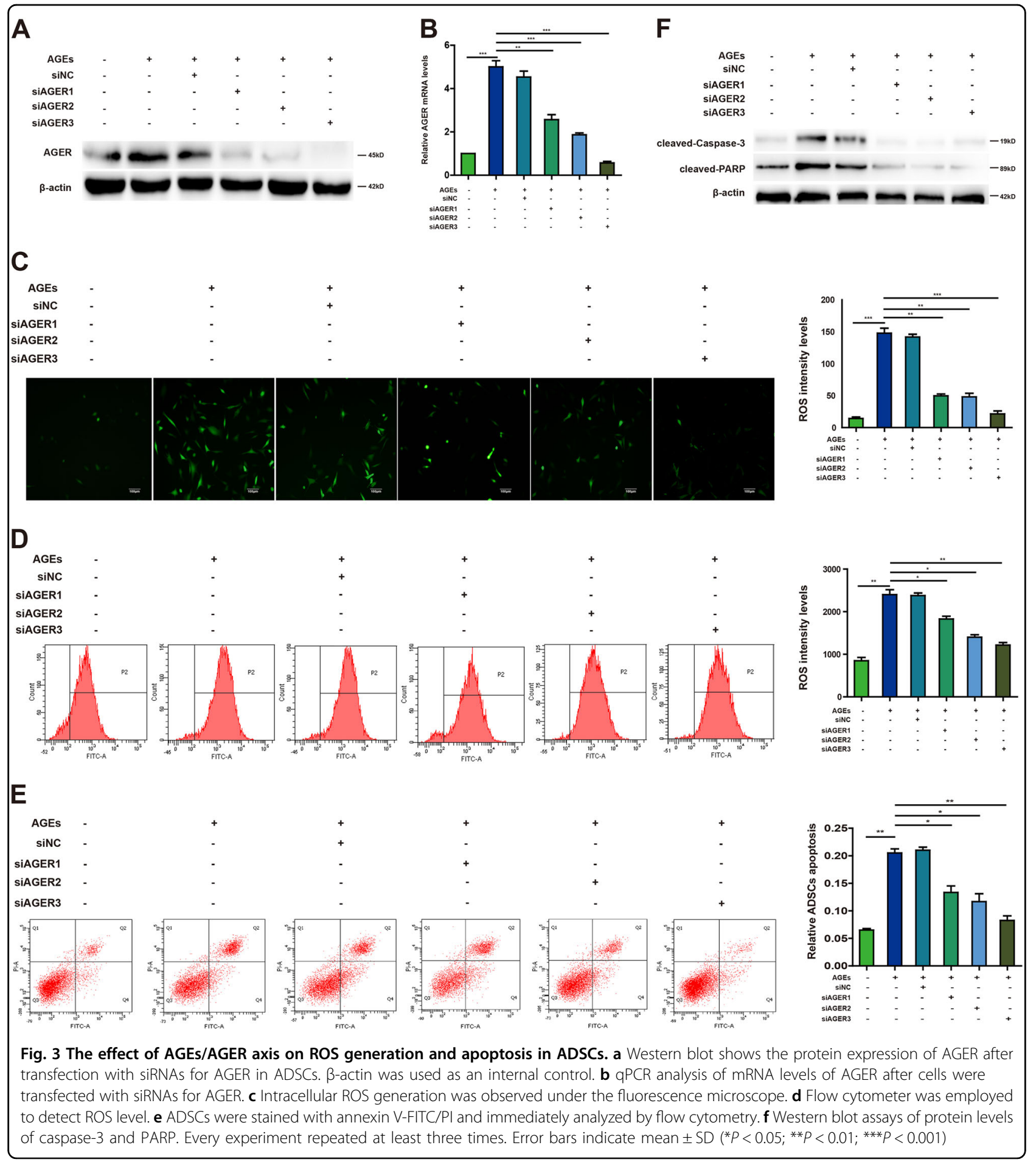

$\mu \mathrm{g} / \mathrm{ml})$. As shown in Figure $6 \mathrm{c}$, both AGEs and anisomycin treatment induced JNK phosphorylation, resulted in high expression of cleaved-caspase- 3 and PARP, but this phosphorylation could be blocked by siRNA for AGER. In addition, knockdown AGER also inhibited caspase-3 pathway, blocked AGEs or anisomycin-induced upregulation of ROS generation and apoptosis of ADSCs
(Fig. 6d-f). Moreover, we employed JNK inhibitor SP600125 to confirm the role of JNK activity in this process. Data from supplementary Figure 2 showed that SP600125 significantly inhibited ROS generation and apoptosis, which was induced by AGEs. So, these results suggested that JNK pathway was required in AGEs/ AGER-mediated ROS generation and cell apoptosis. 


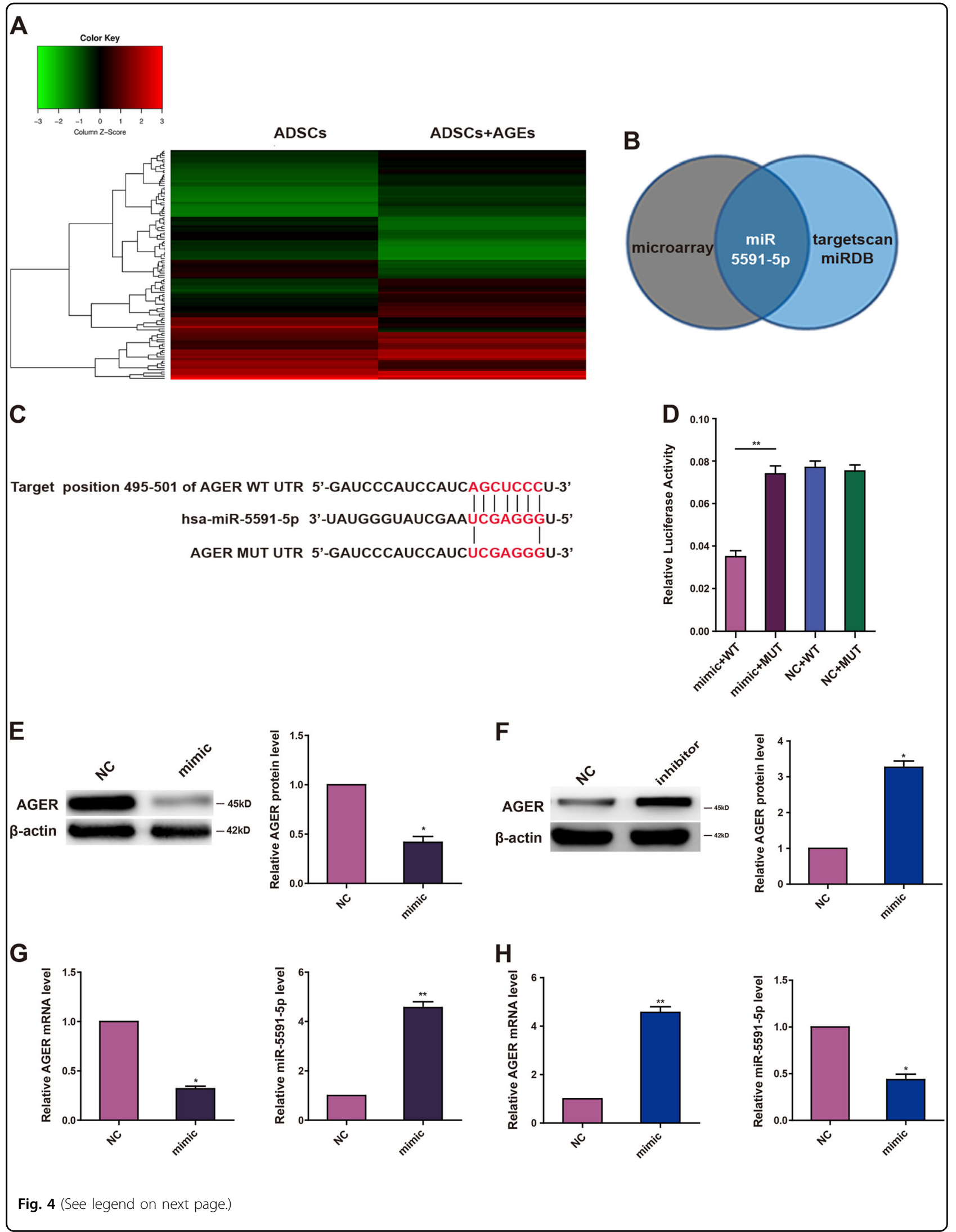


(see figure on previous page)

Fig. 4 AGER is a target of miR-5591-5p. a A heat map showing differentially regulated miRNAs in ADSCs treated with or without AGEs. A color scale is shown below. $\mathbf{b}$ Schema for identification of the putative miRNA(s) that could be downregulated when cells were given AGEs and partly complementary to a conserved site within the $3^{\prime}$ untranslated region of AGER. c The predicted site of miR-5591-5p binding to the AGER $3^{\prime}-U T R$ was detected using bioinformatics tools. $\mathbf{d}$ Mutated site in the AGER $3^{\prime}$-UTR is shown. The effect of miR-5591-5p on luciferase activity induced by the psiCHECK ${ }^{\mathrm{TM}}$-2-AGER-WT and psiCHECK ${ }^{\mathrm{T}}$-2-AGER-MUT reporter plasmids in ADSCs. e, $\mathbf{f}$ Western blot images showing the protein expression levels of AGER when ADSCs transfected with miR-5591-5p mimics and inhibitors. $\mathbf{g}, \mathbf{h}$ qPCR analysis of mRNA levels of miR-5591-5p and AGER after cells were transfected with miR-5591-5p mimic and inhibitor. Data are shown as means \pm SD of three replicates $\left({ }^{*} P<0.05 ;{ }^{* *} P<0.01\right)$

JNK signal is involved in the inhibitory effect of miR-5591$5 p$ on AGEs/AGER-induced ROS generation and apoptosis in ADSCS

Since JNK signal is responsible for the regulation of ROS generation and apoptosis mediated by AGEs/AGER, we wanted to identify whether miR-5591-5p could influence the activity of JNK induced by AGEs/AGER in ADSCs. From Fig. 7a, we can see that although AGEs or anisomycin treatment resulted in phosphorylation of JNK, this was overturned by miR-5591-5p mimics. Moreover,

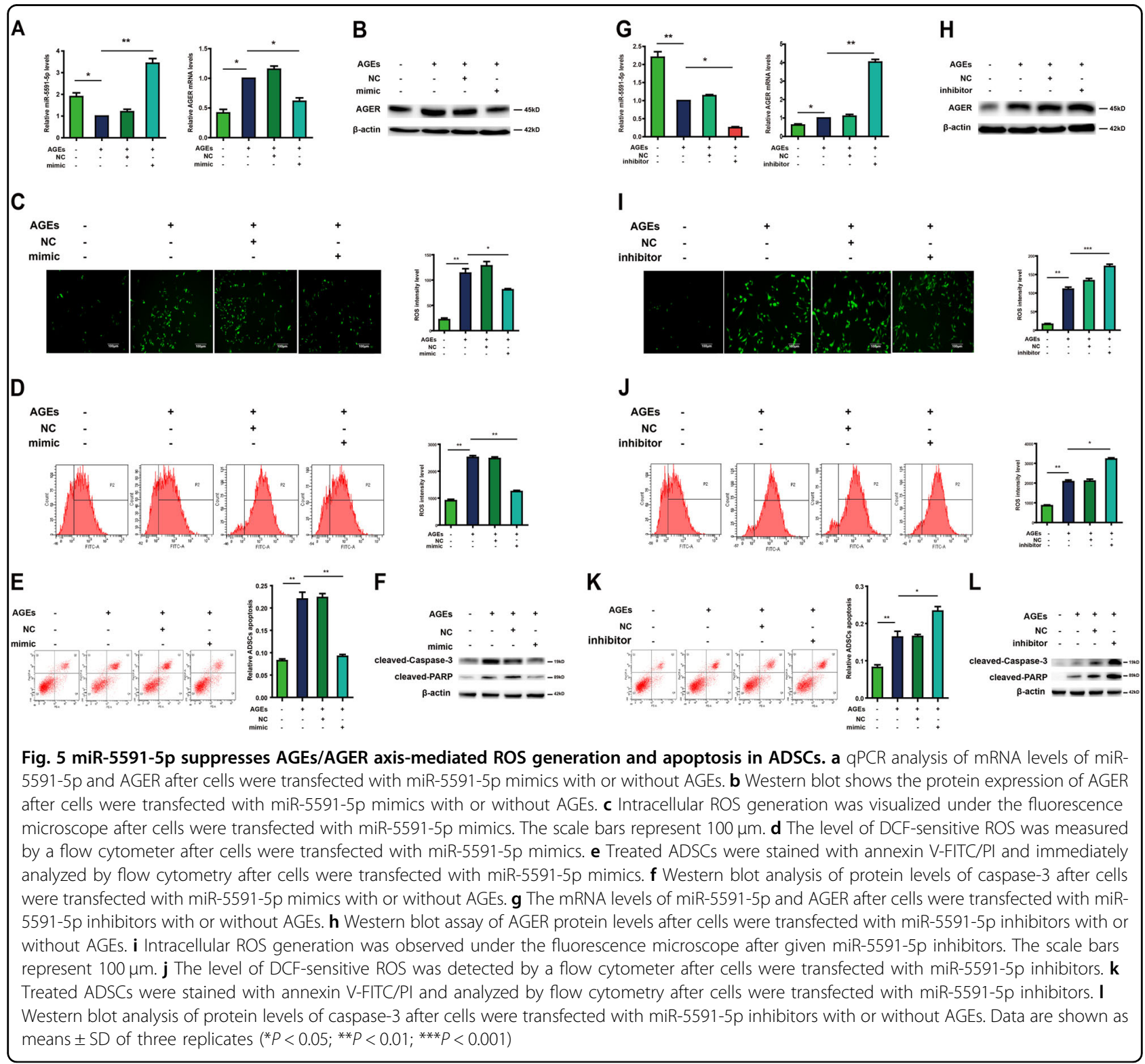



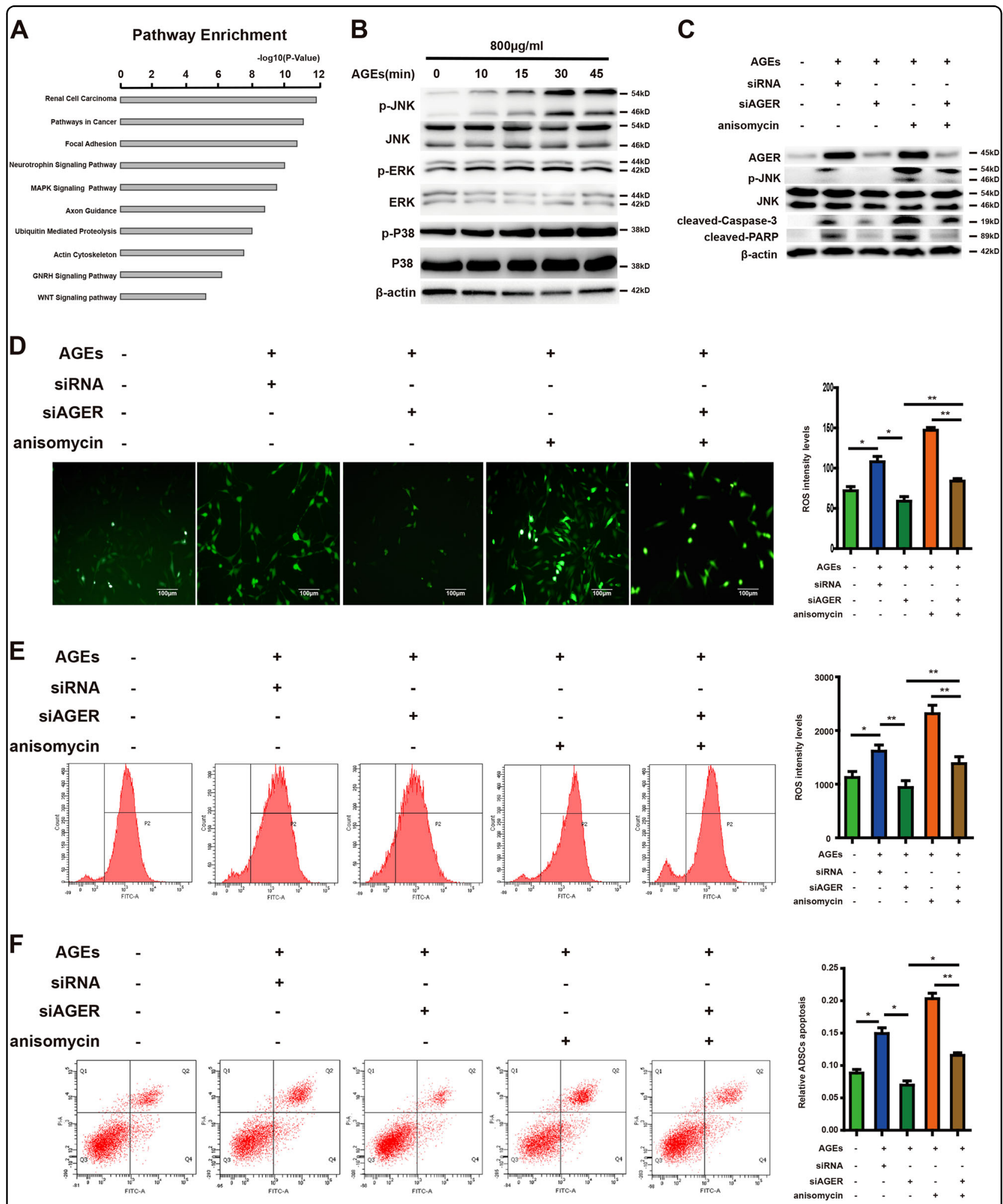

Fig. 6 Role of JNK signal in AGEs/AGER-induced ROS generation and apoptosis in ADSCs. a Top 10 pathways that are enriched for the AGES targets. b Time course analysis of phosphorylated and total p38, ERK, and JNK protein expression in ADSCs treated with AGEs (800 $\mu \mathrm{g} / \mathrm{ml})$. c ADSCs pretreated with AGEs were given AGER siRNA and JNK activator, anisomycin $(1 \mu \mathrm{g} / \mathrm{ml})$. The protein levels of phosphorylated and total JNK, AGER, cleaved-caspase-3 cleaved-PARP, and $\beta$-actin were determined by western blot analysis. $\mathbf{d}$ Intracellular ROS generation was visualized under the fluorescence microscope. e Flow cytometer was employed to detect ROS level. $\mathbf{f}$ Treated ADSCs were stained with annexin V-FITC/PI and immediately analyzed by flow cytometry. Each value is expressed as the mean \pm SD of three independent experiments $\left({ }^{*} P<0.05 ;{ }^{* *} P<0.01 ;{ }^{* * *} P<0.001\right)$ 


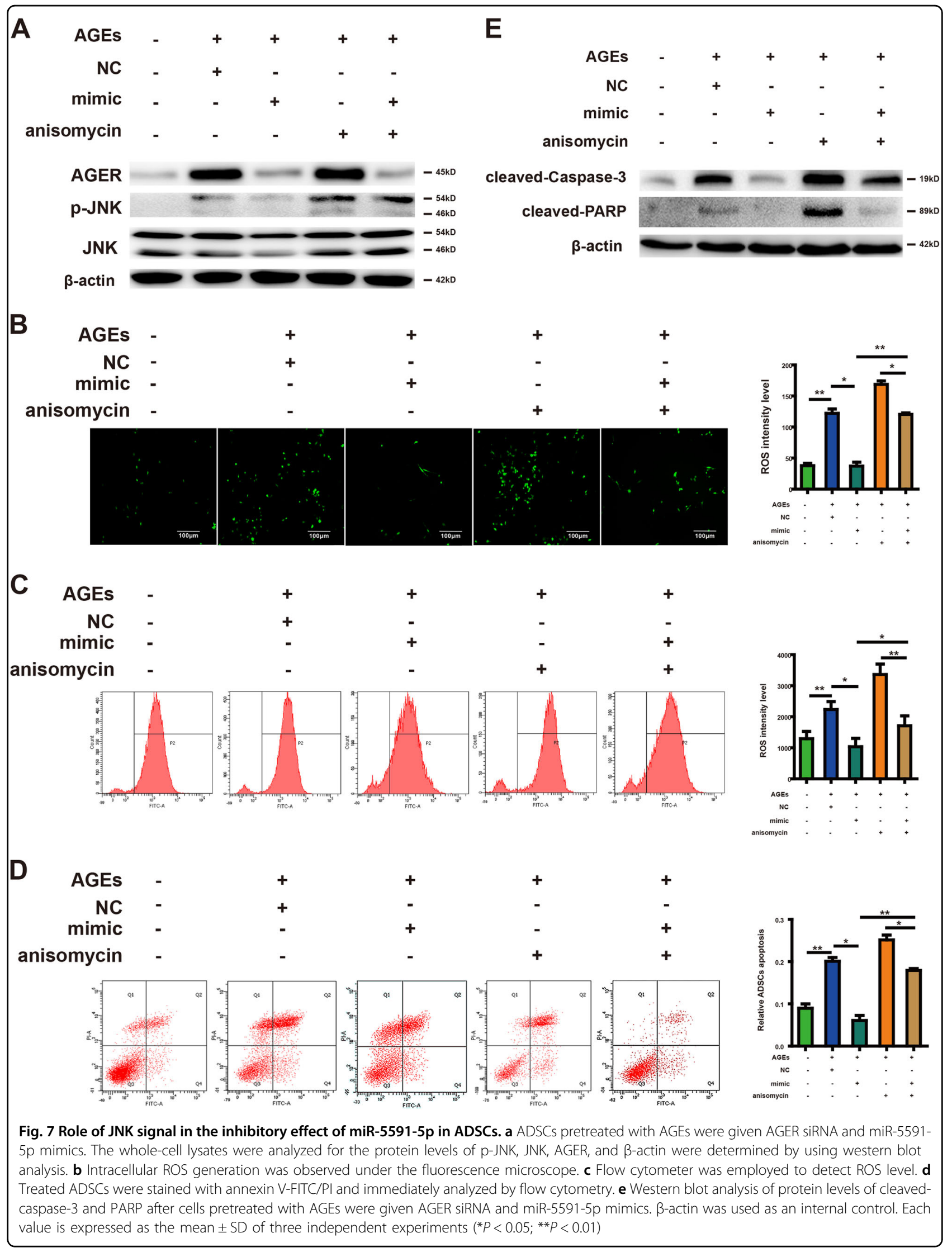




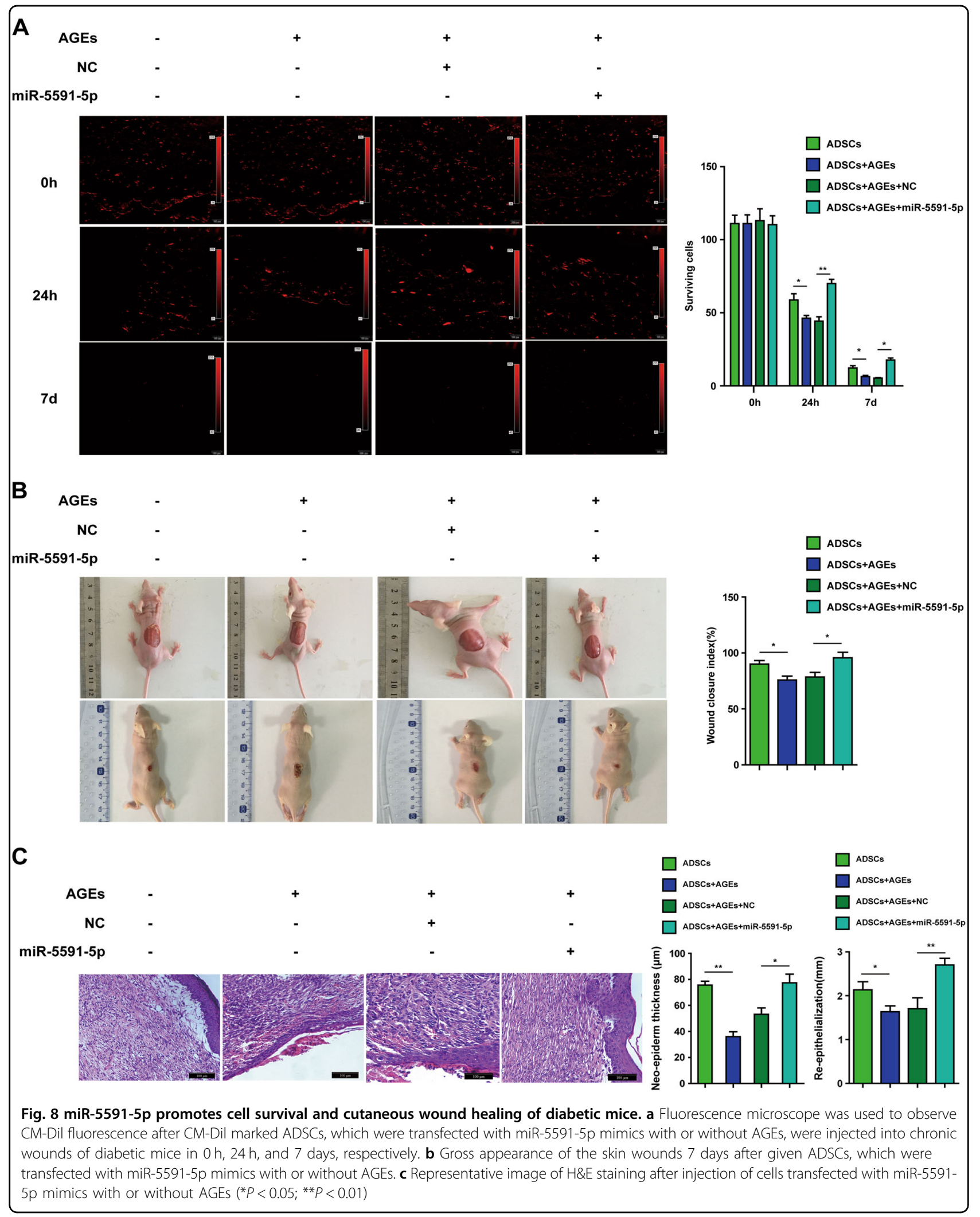


miR-5591-5p mimics suppressed the positive effect of AGEs or anisomycin on ROS generation (Fig. 7b, c). The apoptotic ADSCs were also decreased by miR-5591-5p mimics through activated caspase pathway (Fig. $7 \mathrm{~d}$, e). So, we think that miR-5591-5p may suppress JNK signal activity, which lead to ROS generation and apoptosis in ADSCs.

miR-5591-5p promotes cell survival and cutaneous wound healing in vivo

To determine the effect of miR-5591-5p on cutaneous wound healing in vivo, we first used CM-Dil marked ADSCs to inject into the chronic wounds of diabetic mice. Only a few cells pretreated with AGEs survived in the wound bed. However, more cells expressing miR-5591-5p could be found surviving in the wound bed (Fig. 8a).

We further explore whether forced-expression of miR5591-5p in ADSCs can be prone to the wound healing in diabetic mice. As displayed in Figure 8b, the wound area of diabetic mice was significantly greater when ADSCs pretreated with AGEs. However, ADSCs with miR-5591$5 p$ showed smaller wound area when compared with ADSCs or AGEs pretreated ADSCs, the wound closure index was significantly better than that in AGEs groups (Fig. 9).

In addition, hematoxylin and eosin (H\&E) staining indicated that AGEs-pretreating significantly impair the repairing ability of ADSCs. However, ADSCs with miR5591-5p showed greater repairing ability by promoting epithelization of the wound. The thickness of neoepiderm and re-epithelization in the miR-5591-5p group were significantly greater than that in the control group $\left(0.8^{\circ} \mathrm{C}\right)$.

\section{Discussion}

Although recent studies have shown beneficial effects of ADSCs administration for various diseases, impairment of resident and recruited cell functions strongly results in delays in wound healing under diabetic conditions. Extensive research has showed the vital role of diabetes in regulating stem cell apoptosis in the pathogenesis of delayed wound healing ${ }^{22,}{ }^{23}$. However, the mechanism of diabetes in the damage of ADSCs functions in repairing wound healing has been not elucidated.

AGEs are noxious metabolic products that accumulate in diabetic patients but not in normal individuals. Excessive formation of AGEs is considered as the most important mechanism that triggers the pathophysiological cascades associated with the onset of diabetic complications. There has been evidence that the elevated AGEs in diabetic patients bring pathological damages, such as promotion of EPC and endothelial cell apoptosis, thus impedes wound healing ${ }^{8-10}$. AGER is widely viewed as the best-characterized AGEs receptor, which is responsible

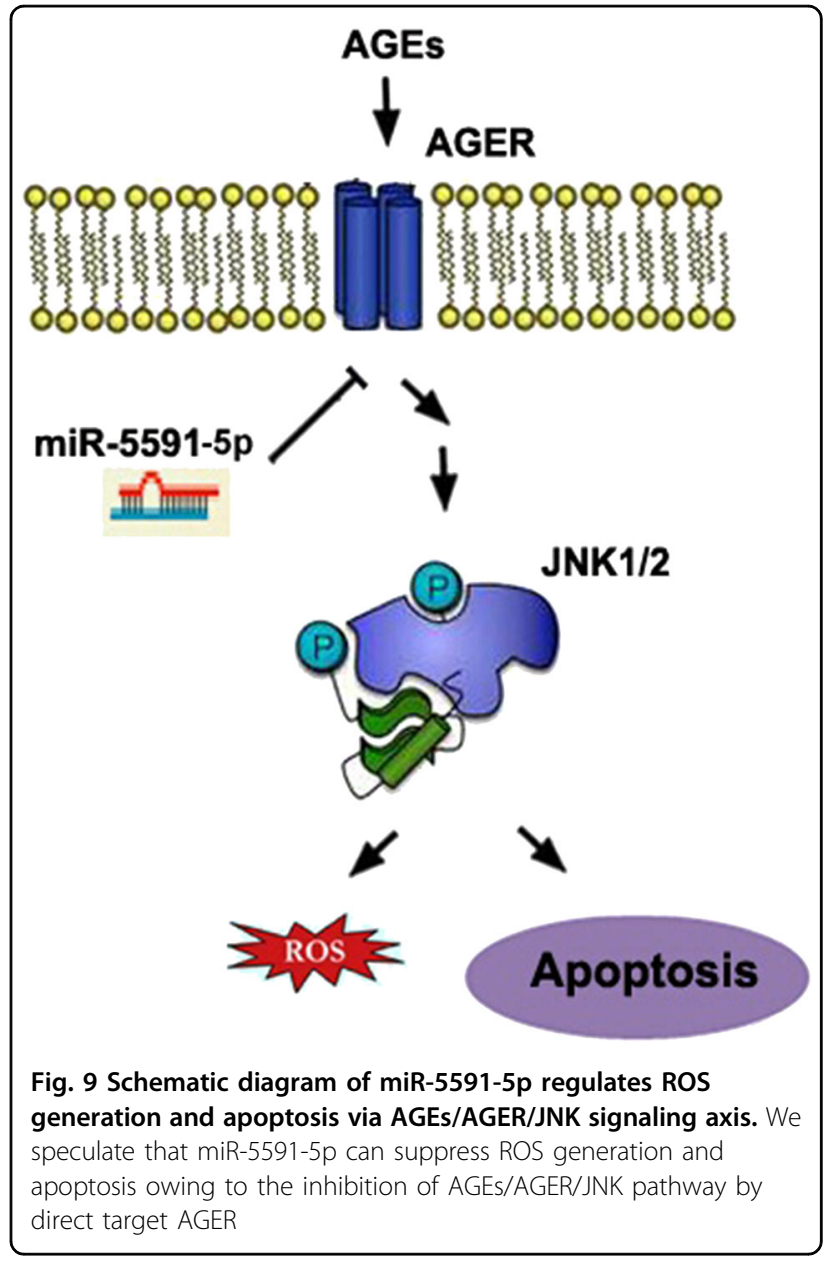

for most of the negative effects of AGEs. Extensive research has suggested that intracellular AGE signaling is mediated via AGER present in most cell types ${ }^{13}, 14$. Apoptosis is a potential mechanism through which AGEs exert its effects on cellular dysfunction ${ }^{24}$. Excessive production of ROS plays a critical role in the process of apoptosis $^{25}$. Previous studies indicate that AGEs/AGER interaction triggers ROS generation and activates downstream JNK pathways and induce apoptosis in $\mathrm{EPCs}^{26}$. AGEs/AGER signaling have been demonstrated to induce ROS production and apoptosis via the JNK and p38 MAPK pathways in osteoblasts and fibroblasts ${ }^{15,} 27$. However, few studies about the AGEs/AGER signaling in ADSCs has been reported. One recent study has demonstrated that AGEs induce apoptosis of ADSCs in vitro via AGER-dependent p38 MAPK pathway ${ }^{28}$. Furthermore, they find that AGE-induced apoptosis is suppressed by antioxidants $\mathrm{N}$-acetylcysteine (NAC) and ascorbic acid 2-phosphate (AAP) ${ }^{29}$. In this study, we demonstrated that the incubation of ADSCs with AGEs resulted in significant upregulation of AGER and cell apoptosis. The interaction of AGEs with AGER regulated 
increased production of ROS and leads to apoptosis through caspase- 3 activity.

miRNAs are a group of small, non-coding, and singlestrand RNAs that control gene expression at the posttranscriptional and translational level by matching their seed sequences to complementary sequences in the $3^{\prime}$ UTR of target mRNAs ${ }^{17}$. Previous studies revealed dysregulated miRNA expression under $\mathrm{AGEs}^{30}$. However, AGEs-mediated alteration of miRNA expression in ADSCs has not been well characterized. Using an miRNA microarray, we surveyed the differential expression of miRNAs after AGEs treatment in ADSCs. The results showed that a panel of miRNAs in AGE-treated ADSCs were significantly dysregulated, which may regulate the ROS production and apoptosis of ADSCs under AGEs. Diabetic conditions can regulate miRNA activity. It has been reported that high glucose reduced EZH2 binding to the miR-101 locus, whereas EZH2 overexpression inhibits miR-101 promoter activity in human fetal endothelial cells of the umbilical cord vein ${ }^{31}$. Diabetic conditions induce miR-379 cluster expression, depends on the expression of the host long non-coding RNA transcript from its promoter ${ }^{32}$. Consequently, as metabolic products in diabetic, AGEs likely control miRNAs expression through regulating their promoter activity.

A number of studies have revealed the involvement of miRNAs in regulating intracellular ROS production and apoptosis. miR-210 is considered to induce apoptosis in colorectal cancer cells via a ROS-dependent mechanism $^{33}$. Loss of miR-137 is confirmed to ameliorate highglucose-induced damage in human umbilical vein endothelial cells (HUVECs) by overexpression of AMPK $\alpha 1$, leading to decreasing oxidative stress ${ }^{34}$. However, only a few studies investigate the role of miRNAs in regulating AGE/AGER signaling related to diabetic manifestations and during diabetes. A genomewide study reveals elevation of miR-214 after treating primary monocytes and monocytic cell line (THP-1) with various AGEs. This is also observed in patients with chronic renal failure ${ }^{30}$. In addition, antioxidants NAC and AAP upregulate miR-223 in ADSCs. miR-223 mimics blocked antioxidant inhibition of AGE-induced apoptosis and ROS generation ${ }^{29}$. In the miRNA microarray, we selected miR-5591-5p because it was the only one that was predicted to target AGER. We demonstrated that miR-5591-5p could directly bind to the $3^{\prime}$ UTR of AGER. miR-5591-5p regulated ROS generation and cell apoptosis induced by AGEs/AGER in ADSCs. Furthermore, miR-5591-5p promoted ADSCs survival and improved the repairing ability of ADSCs for cutaneous wound healing of diabetic mice. These findings indicated the important role of miR-5591-5p in regulating the effect of ADSCs in repairing diabetic wound healing via targeting AGEs/AGER.
MAPK signals are frequently over-activated in a variety of disease states. The activated MAPK pathways have been implicated in mediating apoptotic responses. AGEs have been reported to stimulate the activation of MAPK cascades in different cell types ${ }^{16,35,36}$. It has been shown that AGEs induces apoptosis of ADSCs in vitro via AGERdependent p38 MAPK pathway ${ }^{28}$. More and more miRNAs have been identified that modulate MAPK pathway in stem cells. Mei et al. ${ }^{37}$ find that miR-21 promotes adipogenesis and osteogenesis of MSCs through modulating the ERK-MAPK signaling pathway. In addition, miR-126 is considered to promote mesenchymal stem cells differentiation toward endothelial cells via activating PI3K/Akt and MAPK/ERK pathway ${ }^{38}$. To further investigate whether MAPK signals are involved in regulating the effect of ADSCs on repairing diabetic wound healing by miR-5591-5p, we first detected the levels of phosphorylated forms of MAPK family members after treated with AGEs. We found that JNK signal pathway involved in the regulation of ROS generation and apoptosis mediated by AGEs/AGER. Then, we examined whether miR-5591$5 p$ can influence the activity of JNK induced by AGEs/ AGER in ADSCs. Our results revealed that miR-5591-5p may suppress JNK signal activity, which causes ROS generation and apoptosis of ADSCs.

In summary, we have shown that the interaction of AGEs with AGER regulated increased production of ROS and leads to apoptosis of ADSCs. miR5591-5p can regulate the effect of ADSCs in repairing diabetic wound healing via targeting AGES/AGER axis. Moreover, JNK signal is involved in the inhibitory effect of miR-5591-5p on AGEs/AGER-induced ROS generation and apoptosis in ADSCs. Our studies uncover the mechanism of miR5591-5p that regulate specifically the effect of ADSCs in repairing diabetic wound via targeting AGES/AGER/JNK signaling in order to formulate improved and optimized miRNA-targeted approaches for therapy of diabetic wound healing.

\section{Materials and methods}

\section{Isolation and culture of human ADSCs}

All the methods were carried out as described in our previous study ${ }^{4}$. Adipose tissue samples were obtained from three liposuction aspirates of patients with informed consent at the Affiliated Hospital of Xuzhou Medical University. Isolated ADSCs were maintained in L-Dulbecco's modified Eagle's medium (DMEM; Invitrogen, CA, USA) containing 10\% fetal bovine serum (FBS; Invitrogen) in $37^{\circ} \mathrm{C}$ humidifed incubator with $5 \% \mathrm{CO}_{2}$.

\section{Cell treatment}

AGEs were obtained from Sigma-Aldrich (St. Louis, MO, USA). AGEs were added to ADSCs and then subject to some experiments. Also, a JNK activator, anisomycin 
Table 1 Primer sequences

\begin{tabular}{ll}
\hline Name & Primer \\
\hline AGER & Sense: 5'-GGCTGGTGTTCCCAATAAGG-3'; anti-sense: 3'-TCACAGGTCAGGGTTACGGTTC-5' \\
miR-5591-5p & Sense: 5'-GATGCCCATGCCGATTCTT-3'; anti-sense: 3'-ACTGGGCTTCGATTTCACCT-5' \\
miR-5591-5p inhibitor & Sense: 5'-GATGCCCATGCCGATTCTT-3'; anti-sense: 3'-ACTGGGCTTCGATTTCACCT-5' \\
\hline
\end{tabular}

$(0.1 \mu \mathrm{g} / \mathrm{ml}$, Beyotime, Shanghai, China) was used to activate the JNK signaling pathway. A JNK inhibitor, SP600125 (20 $\mu \mathrm{g} / \mathrm{ml}$, Beyotime, Shanghai, China) was added to cultured cells to block the JNK activity in this study.

\section{Cell transfection}

The AGER siRNAs were purchased from GenePharma (Shanghai, China). The sequences for the AGER siRNA used for the experiments were as follows:

AGER siRNA1: 5'-GCCAACCCAGAAGCTAGAA -3'; AGER siRNA2: 5' - GTGAATCCTGCCTCTGAAC-3'; AGER siRNA3: 5' - GCCTCTGAACTCACAGCCA-3'.

ADSCs were plated in six-well or 96-well plates and transient transfection using Lipofectamine2000 (Invitrogen) according to the manufacturer's instructions.

Stable transfection for cells was performed as previously described $^{39}$. LV5-Control and LV5-miR-5591-5p lentiviral vectors were purchased from Shanghai GenePharma Company (China). Lentiviruses mixed with polybrene (5 $\mathrm{mg} / \mathrm{ml}$ ) were added to ADSCs and puromycin $(5 \mathrm{mg} / \mathrm{ml})$ was applied to select positive clones. Stable miR-5591-5p transfectants were isolated after 2 weeks.

\section{RNA extraction and qPCR}

Total RNA was extracted and complementary DNA was generated as previously described ${ }^{40}$. qPCR was employed on a 7500 HT Fast qPCR instrument (New York, USA) as previously described $^{40}$. The primer sets were shown in Table 1.

\section{Measurement of intracellular ROS}

Cells were seeded in a six-well plate plated with $5 \times 10^{4}$ cells per well. Cells were added with $10 \mu \mathrm{M}$ fluorescent probe CM-H2DCFDA (Molecular Probes; Invitrogen), incubated for $60 \mathrm{~min}$ at $37^{\circ} \mathrm{C}$. After washing with PBS, cells were detected using fluorescence microscope (Olympus, Tokyo, Japan) and FACSCalibur flow cytometer (BD Biosciences, Franklin Lakes, NJ, USA).

\section{Apoptosis assay}

Following treatment, ADSCs were stained with fluorescein (FITC)-conjugated annexin $\mathrm{V}$ and propidium iodide (FITC/PI) (KeyGen Biotech, Nanjing, China), and analyzed on flow cytometer to determine rate of apoptosis.

\section{Luciferase reporter gene assay}

ADSCs were transfected with either psiCHECK-2/ AGER 3'-UTR, miR-5591-5p mimics or control mimics, and then subjected to luciferase assay using the dualluciferase reporter assay kit (Promega, Madison, WI, USA) according to instructions.

\section{Western blot}

Cell extracts were separated on SDS-polyacrylamide gel, then proteins were transferred to a nitrocellulose membrane and incubated with the following antibodies: rabbit polyclonal against AGER (1:500; Santa Cruz, CA, USA), cleave-caspase 3 (1:800), cleave-PARP (1:800), JNK (1:800), p-JNK (1:500), ERK (1:800), and p-ERK (1:1000), p38 (1:500), p-p38 (1:500), and rabbit monoclonal against $\beta$ actin (1:1000; Cell Signaling Technology, Danvers, MA, USA). Immunoreactive protein bands were detected with Tanon scanning system.

\section{Diabetic wound model}

Six-week-old nu/nu athymic nude mice were purchased from Vital River (Beijing, China) and maintained under specific pathogen-free conditions. All animal studies were approved by Animal Care and Use Committee. Diabetic mice were induced by intraperitoneal injection of $150 \mathrm{mg} /$ kg STZ (Sigma, Shanghai, China). Blood glucose level in tail venous blood samples was measured 14 days later. Animals with blood glucose levels above $300 \mathrm{mg} / \mathrm{dl}$ and apparent features of polydipsia, polyphagia, and polyuria were considered to be diabetic. After anesthetizing animals with intraperitoneal injections of chloral hydrate $(10 \%, 5 \mathrm{ml} / \mathrm{kg})$, a full thickness skin defect of $2-\mathrm{cm}$ diameter was made on the back. The wound area was analyzed as previously described ${ }^{4}$.

\section{ADSCs survival assay in vivo}

ADSCs were marked with CM-Dil prior to injection. When ADSCs after the third passage reached 70-80\% confluency, they were incubated with $5 \mu \mathrm{g} / \mathrm{ml} \mathrm{CM-Dil} \mathrm{at}$ $37^{\circ} \mathrm{C}$ for $30 \mathrm{~min}$ and then washed with PBS three times. The efficiency of the CM-Dil staining was detected with a 
fluorescence microscope. Following treatment, $1 \times 10^{6}$ ADSCs were injected around the wound bed. At $0 \mathrm{~h}, 24 \mathrm{~h}$, and 7 days after transplantation, under anesthesia the wounds were collected with the surrounding tissue. The skin tissues were embedded in OCT compound (TissueTek ${ }^{\circ}$, Miles Laboratories, Naperille, IL, USA) at $-80^{\circ} \mathrm{C}$. CM-Dil-labeled ADSCs were observed to examine CMDil fluorescence as whole mounts under a fluorescence microscope with excitation at $420 \mathrm{~nm}$ and emission at $480 \mathrm{~nm}$ (Olympus, Tokyo, Japan).

\section{H\&E staining}

Seven days after transplantation, the wounds with the surrounding tissue were collected and fixed in $10 \%$ formalin, and H\&E staining was performed to observe the epithelization ${ }^{41}$. Images were collected by a Camedia Master C-3040 digital camera (Olympus).

\section{Statistical analysis}

Results were presented as mean \pm SD and statistically analyzed utilizing a Student's $t$-test with SPSS software (SPSS 16.0, Inc., Chicago, IL, USA). $P<0.05$ was considered as a statistically significant difference.

\section{Acknowledgements}

This work was supported by grants from the National Natural Science Foundation of China (nos. 81501671 and 81571901).

\section{Authors' contributions}

Q.L., S.X., and Y.Y. conducted the experiments. Q.L. and Y.Y. analyzed the results. Q.L., S.X., and F.C. wrote the manuscript. F.C. and P.J. conceived the experiments. All authors reviewed the manuscript.

\section{Conflict of interest}

The authors declare that they have no conflict of interest.

\section{Publisher's note}

Springer Nature remains neutral with regard to jurisdictional claims in published maps and institutional affiliations.

Supplementary Information accompanies this paper at (https://doi.org/ 10.1038/s41419-018-0615-9).

Received: 10 December 2017 Revised: 3 April 2018 Accepted: 18 April 2018 Published online: 11 May 2018

\section{References}

1. Konno, M. et al. Adipose-derived mesenchymal stem cells and regenerative medicine. Dev. Growth Differ. 55, 309-318 (2013).

2. Locke, M., Feisst, V. \& Dunbar, P. R. Concise review: human adipose-derived stem cells: separating promise from clinical need. Stem Cells 29, 404-411 (2011).

3. Cianfarani., F. et al. Diabetes impairs adipose tissue-derived stem cell function and efficiency in promoting wound healing. Wound Repair Regen. 21, 545-553 (2013).

4. Li, Q., Guo, Y., Chen, F. F., Liu, J. \& Jin, P. S. Stromal cell-derived factor-1 promotes human adipose tissue-derived stem cell survival and chronic wound healing. Exp. Ther. Med. 12, 45-50 (2016).
5. Ohmura., Y. et al. Combined transplantation of pancreatic islets and adipose tissue-derived stem cells enhances the survival and insulin function of islet grafts in diabetic mice. Transplantation 90, 1366-1373 (2010).

6. Saki, N., Jalalifar, M. A., Soleimani, M., Hajizamani, S. \& Rahim, F. Adverse effect of high glucose concentration on stem cell therapy. Int. J. Hematol. Oncol. Stem Cell Res. 7, 34-40 (2013).

7. Goldin, A., Beckman, J. A., Schmidt, A. M. \& Creager, M. A. Advanced glycation end products: sparking the development of diabetic vascular injury. Circulation 114, 597-605 (2006).

8. Ge, K. et al. Influence of advanced glycosylation end products on wound healing of burn rats with diabetes. Chin. J. Burns 25, 433-436 (2009).

9. Sun., C. et al. Advanced glycation end products depress function of endothelial progenitor cells via p38 and ERK 1/2 mitogen-activated protein kinase pathways. Basic Res. Cardiol. 104, $42-49$ (2009).

10. Pan, Y. et al. Platelet-secreted microRNA-223 promotes endothelial cell apoptosis induced by advanced glycation end products via targeting the insulin-like growth factor 1 receptor. J. Immunol. 192, 437-446 (2014).

11. Barlovic, D. P., Thomas, M. C. \& Jandeleit-Dahm, K. Cardiovascular disease: what's all the AGE/RAGE about? Cardiovasc. Hematol. Disord. Drug Targets 10, 7-15 (2010).

12. Kim, S. et al. COMP-Ang1 inhibits apoptosis as well as improves the attenuated osteogenic differentiation of mesenchymal stem cells induced by advanced glycation end products. Biochim. Biophys. Acta 1830, 4928-4934 (2013).

13. Zong, H., Ward, M. \& Stitt, A. W. AGES, RAGE, and diabetic retinopathy. Curr. Diab. Rep. 11, 244-252 (2011).

14. Ramasamy, R. et al. Advanced glycation end products and RAGE: a common thread in aging, diabetes, neurodegeneration, and inflammation. Glycobiology 15, 16-28 (2005).

15. Alikhani, M. et al. Advanced glycation end products stimulate osteoblast apoptosis via the MAP kinase and cytosolic apoptotic pathways. Bone $\mathbf{4 0}$ 345-353 (2007)

16. Hsu, W. H. et al. Berberine induces apoptosis in SW620 human colonic carcinoma cells through generation of reactive oxygen species and activation of JNK/p38 MAPK and FasL. Arch. Toxicol. 81, 719-728 (2007).

17. Ambros, V. microRNAs: tiny regulators with great potential. Cell $\mathbf{1 0 7}, 823-826$ (2007).

18. Lee, C. Y. et al. MicroRNA-mediated down-regulation of apoptosis signal-regulating kinase 1 (ASK1) attenuates the apoptosis of human mesenchymal stem cells (MSCs) transplanted into infarcted heart. Int. J. Mol. Sci. 17, 10 (2016).

19. $\mathrm{Wu}, \mathrm{Q}$. et al. Advanced oxidation protein products induce chondrocyte apoptosis via receptor for advanced glycation end products-mediated, redoxdependent intrinsic apoptosis pathway. Apoptosis 21, 36-50 (2016).

20. Kim, J. H. et al. A miRNA-101-3p/Bim axis as a determinant of serum deprivation-induced endothelial cell apoptosis. Cell Death Dis. 8, e2808 (2017).

21. Togliatto, G. et al. Unacylated ghrelin induces oxidative stress resistance in a glucose intolerance and peripheral artery disease mouse model by restoring endothelial cell miR-126 expression. Diabetes 64, 1370-13782 (2015).

22. Albiero, M. et al. Defective recruitment, survival and proliferation of bone marrow-derived progenitor cells at sites of delayed diabetic wound healing in mice. Diabetologia 54, 945-953 (2011).

23. Ko, K. I. et al. Diabetes reduces mesenchymal stem cells in fracture healing through a TNFalpha-mediated mechanism. Diabetologia 58, 633-642 (2015).

24. Weinberg, E., Maymon, T. \& Weinreb, M. AGEs induce caspase-mediated apoptosis of rat BMSCs via TNFalpha production and oxidative stress. J. Mol. Endocrinol. 52, 67-76 (2014).

25. Ullah, I., Park, H. Y. \& Kim, M. O. Anthocyanins protect against kainic acidinduced excitotoxicity and apoptosis via ROS-activated AMPK pathway in hippocampal neurons. CNS Neurosci. Ther. 20, 327-338 (2014).

26. Chen, J. et al. Advanced glycation endproducts induce apoptosis of endothelial progenitor cells by activating receptor RAGE and NADPH oxidase/JNK signaling axis. Am. J. Transl. Res. 8, 2169-2178 (2016).

27. Alikhani, M. et al. Advanced glycation end products induce apoptosis in fibroblasts through activation of ROS, MAP kinases, and the FOXO1 transcription factor. Am. J. Physiol. Cell Physiol. 292, 850-856 (2007).

28. Wang, Z et al. Effect of advanced glycosylation end products on apoptosis in human adipose tissue-derived stem cells in vitro. Cell Biosci. 5, 3 (2015).

29. Wang, Z., Li, H., Guo, R., Wang, Q. \& Zhang, D. Antioxidants inhibit advanced glycosylation end-product-induced apoptosis by downregulation of miR-223 in human adipose tissue-derived stem cells. Sci. Rep. 6, 23021 (2016). 
30. Li, L. M. et al. Role of microRNA-214-targeting phosphatase and tensin homolog in advanced glycation end product-induced apoptosis delay in monocytes. J. Immunol. 186, 2552-2560 (2011).

31. Floris, I. et al. Gestational diabetes mellitus impairs fetal endothelial cel functions through a mechanism involving microRNA-101 and histone methyltransferase enhancer of zester homolog-2. Arterioscler. Thromb. Vasc. Biol. 35, 664-674 (2015).

32. Kato, M. et al. An endoplasmic reticulum stress-regulated IncRNA hosting a microRNA megacluster induces early features of diabetic nephropathy. Nat. Commun. 7, 12864 (2016).

33. Tagscherer, K. E. et al. MicroRNA-210 induces apoptosis in colorectal cancer via induction of reactive oxygen. Cancer Cell Int. 16, 42 (2016).

34. Li, J., Li, J., Wei, T. \& Li, J. Down-regulation of microRNA-137 improves high glucose-induced oxidative stress injury in human umbilical vein endothelial cells by up-regulation of AMPKalpha1. Cell. Physiol. Biochem. 39, 847-859 (2016).

35. Yin, Y., Liu, W., Ji, G. \& Dai, Y. The essential role of p38 MAPK in mediating the interplay of oxLDL and IL-10 in regulating endothelial cell apoptosis. Eur. J. Cell Biol. 92, 150-159 (2013)

36. Xu, F. Zhang, C. \& Graves, D. T. Abnormal cell responses and role of TNF-alpha in impaired diabetic wound healing. Biomed. Res. Int. 2013, 754802 (2013)
37. Mei, Y. et al. miR-21 modulates the ERK-MAPK signaling pathway by regulating SPRY2 expression during human mesenchymal stem cell differentiation. J. Cell. Biochem. 114, 1374-1384 (2013).

38. Huang, F. et al. Overexpression of miR-126 promotes the differentiation of mesenchymal stem cells toward endothelial cells via activation of PI3K/Akt and MAPK/ERK pathways and release of paracrine factors. Biol. Chem. 394 1223-1233 (2013)

39. Jing, R. et al. Plasma miR-185 is decreased in patients with esophageal squamous cell carcinoma and might suppress tumor migration and invasion by targeting RAGE. Am. J. Physiol. Gastrointest. Liver Physiol. 309, G719-G729 (2015).

40. Shao, Y., LV, C., Wu, C., Zhou, Y. \& Wang, Q. Mir-217 promotes inflammation and fibrosis in high glucose cultured rat glomerular mesangial cells via Sirt1/HIF-1alpha signaling pathway. Diabetes Metab. Res. Rev. 32, 534-543 (2016).

41. $\mathrm{Wu}$, J. et al. Comparative study of heparin-poloxamer hydrogel modified bFGF and aFGF for in vivo wound healing efficiency. ACS Appl. Mater. Interfaces 8 , 18710-18721 (2016). 